\title{
Lessons from the management of chronic heart failure
}

\section{T A McDonagh}

Heart 2005;91(Suppl II):ii24-ii27. doi: 10.1136/hrt.2005.062067

In seeking to implement evidence based medicine for the patient with heart failure occurring affer a myocardial infarction (MI), much can be learnt from the long road to delivery of best care for the patient with chronic heart failure (CHF) caused by left ventricular systolic dysfunction. Both patient groups are part of the same cardiovascular continuum. A mass of evidence has accrued for the beneficial effects of angiotensin converting enzyme inhibitors, $\beta$ blockers, and aldosterone antagonists on both morbidity and mortality across a wide spectrum of patient severity. This evidence has informed the development of management guidelines, although registry data showed that uptake of treatments remained low, leading to research focused on how heart failure care could be delivered more effectively. This has resulted in a range of heart failure management programmes, many of which have been shown to reduce hospital admission rates and to improve adherence with treatments. Multidisciplinary heart failure management programmes that span primary and secondary care are now considered a routine "standard" to be aspired to in delivering effective CHF care. Applying such an approach to the care of the post-MI heart failure patient should be equally important.

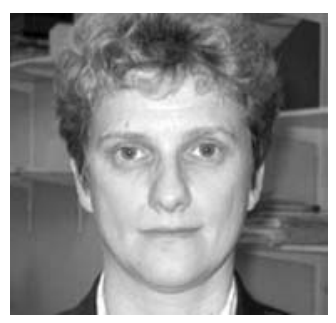

C ardiologists working in heart failure are fortunate in having almost 20 years of evidence from randomised controlled trials to guide their practice. These trials have proven the efficacy of angiotensin converting enzyme (ACE) inhibitors, $\beta$ blockers and aldosterone antagonists, and have informed clinical practice guidelines published by internationally recognised bodies such as the European Society of Cardiology (ESC). ${ }^{1}$ However, registry data suggest that the uptake of evidence based treatments in the community is low while mortality and morbidity remain high. This requires a shift in focus to look again not at what treatments patients are being giving but at how systems of care delivery can improve uptake of treatment for heart failure. This has particularly pertinent lessons which can be applied to the management of post-myocardial infarction (MI) heart failure and left ventricular dysfunction.

Recent data from the EuroHeart failure survey, which looked at in-patients with a discharge diagnosis of heart failure from all over Europe, suggest that clinicians are now doing better with regard to ACE inhibitors: among patients with systolic dysfunction with a diagnosis of heart failure (the group for which there is the most evidence), nearly $80 \%$ of patients are getting an ACE inhibitor and 6\% an angiotensin II receptor antagonist. ${ }^{2}$ However, at less than 50\%, the uptake of $\beta$ blockers throughout Europe is still low.

In primary care, the IMPROVEMENT of heart failure programme tells a similar story: there has been a reasonable uptake of ACE inhibitors at over $70 \%$ but a very low uptake of $\beta$ blockers in patients with heart failure. ${ }^{3}$ Yet, even where good rates of prescriptions of these drugs are being achieved, the target doses of both ACE inhibitors and $\beta$ blockers are only about $50 \%$ of those recommended in ESC guidelines.

Data from the national registry of myocardial infarction in the USA suggest that the situation is no better for myocardial infarction. In a subgroup analysis that looked at more than 600000 cases of myocardial infarction in patients with no history of heart failure between 1994 and 2000, about 20\% of patients had heart failure at presentation and a further $8.6 \%$ developed heart failure during admission. ${ }^{4}$ However, among those with heart failure at discharge, ACE inhibitor usage is less than 40\% while $\beta$ blocker usage is similarly low. A patient with a myocardial infarction complicated by heart failure needs a $\beta$ blocker, yet seems to be less likely to get it in the post-MI setting than in the chronic heart disease situation. Why should this be?

\section{CHF: A COMPLEX DISEASE TO MANAGE}

The management of heart failure is complex. Heart failure is difficult to diagnose; indeed, heart failure is not a diagnosis itself but a syndrome, which means that clinicians must look for a number of aetiologies to see if anything can be reversed. Patients frequently have a number of co-morbidities either caused by the disease or their medication, and the treatment is exceedingly complex. A further reason for the problem may lie with the patient. Where clinical trials have tended to recruit relatively young male patients, the typical heart failure patient is just as likely to be female, while the median age of presentation of heart failure in the UK and much of Europe is 76 years of age, which

Abbreviations: $A C E$, angiotensin converting enzyme; CAPRICORN, carvedilol post-infarct survival control in left ventricular dysfunction; CHAMP, cardiac hospitalisation atherosclerosis management program; CHF, chronic heart failure; ESC, European Society of Cardiology; GRACE, global registry of acute coronary events; IMPACT, initiation management predischarge: process for assessment of carvedilol therapy in heart failure; MI, myocardial infarction 


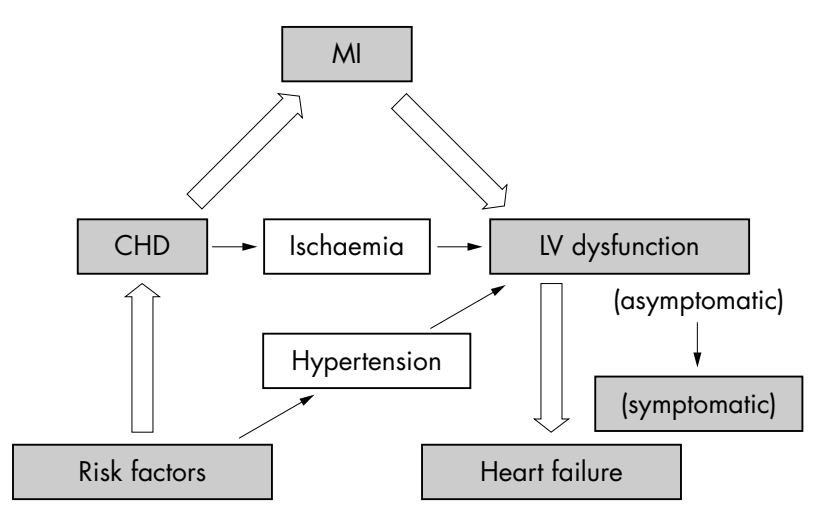

Figure 1 Most patients who develop heart failure, do so following a myocardial infarction and development of left ventricular dysfunction. CHD, coronary heart disease; LV, left ventricular; MI, myocardial infarction.

has led to the description of heart failure as a "cardiogeriatric syndrome".

In addition, the patient often has multiple co-morbidities-around one third have chronic obstructive pulmonary disease, while recent data suggest that more than one third have worsening renal function at presentation. ${ }^{5}$ On top of this, we treat these patients with multiple drugs, many of which are associated with side effects: we give them diuretics and ask them to restrict fluids, and we expect them to change their diet and other aspects of their lifestyle. Even though it is rarely easy for young patients to change their behaviour, we seem to expect patients of 76 to undertake this momentous task, while at the same time subjecting them to multiple health professionals and tests in a multitude of clinics. It is, perhaps, not surprising that patients end up confused and poorly compliant with treatment.

The solution to this problem is the multidisciplinary team: a lot of professionals are needed to look after this complex problem.

\section{THE MULTIDISCIPLINARY TEAM APPROACH TO HEART FAILURE}

The multidisciplinary approach to the management of heart failure is based on clinical trial evidence dating back to the mid 1990s. ${ }^{6}$ Rich and colleagues randomised patients to either a nurse directed, multidisciplinary intervention or to usual care. The trial included "typical" patients: those admitted to hospital over the age of 70 years with heart failure as a result of an MI, and a history of at least four admissions in the past year. These patients were, therefore, at high risk of re-admission.

The intervention delivered was complex: it included education of the patient, dietary advice, review by a cardiologist of the patient's condition and their medication, extensive home visits, and telephone calls. Even in a relatively short follow up of 90 days after discharge, the multidisciplinary intervention reduced all cause admissions by $44 \%(p=0.035)$ compared with usual care, while heart failure admissions were reduced by $56 \%$.

A subsequent study from Adelaide looked at a different aspect of home based care for heart failure admissions. ${ }^{7}$ The intervention was less intensive and involved a nurse visiting the patient once at home after discharge for heart failure, to educate the patient about their disease and medication. Again in this study, both heart failure admissions and all cause admissions were reduced in those receiving the intervention. In addition, this study also showed a reduction in mortality among those assigned to the multidisciplinary care group.
A study conducted at the Western Infirmary in Glasgow looked at 165 patients admitted with acute heart failure, who were randomised to nurse intervention or usual care. ${ }^{8}$ The nurse intervention was fairly intensive, and involved education of the patient about their disease and its treatment, including training in how to adjust diuretic dosages, home visiting, telephone contact, extensive monitoring of the patient with blood tests, and up-titration of medication by the nurse. The study showed an impressive reduction in the number of all cause admissions in the treatment group ( $86 \mathrm{v}$ $114, \mathrm{p}=0.018$ ), and also in heart failure admissions (19 $\mathrm{v}$ $45, \mathrm{p}<0.001)$. Importantly, the patients assigned to the nurse intervention spent significantly fewer days in hospital ( $3.43 v 7.46$ days, $\mathrm{p}=0.0051$ ). This effect was measured at one year so it seems to be a longer term effect than in some of the previous studies.

Overall, the many randomised studies of multidisciplinary management of heart failure provide a substantial evidence base even though the interventions have differed. Some could be described as being multidisciplinary and then using a heart failure clinic to assess patients, some have employed multidisciplinary care without involving a heart failure clinic, others have concentrated on telephoning the patient and encouraging them to telephone their general practitioner when problems arise, while still others have focused on enhancing patient education before they leave hospital.

A recent systematic review of 29 randomised trials of multidisciplinary care in heart failure ${ }^{9}$ suggests that:

- among those which have involved a specialised multidisciplinary team, whether in a clinic or non-clinic setting, mortality has been reduced by $25 \%$, heart failure hospitalisations have been reduced by $26 \%$, and all cause hospitalisations by $19 \%$

- those that focused just on enhancing patient self care by education reduce hospitalisations for heart failure by around $34 \%$ and all cause hospitalisations by $27 \%$

- those in which patients are given telephone contact and are referred to the general practitioner for further help reduce heart failure hospitalisations by $25 \%$ but do not reduce all cause admissions or mortality.

Overall, multidisciplinary team intervention appears to be associated with a higher use of evidence based treatments and higher doses achieved (in six of the 10 studies that have reported this parameter), while in the six studies that have commented on adherence rates to medication five showed improved adherence. This suggests that higher rates of medication and higher adherence rates seem to be part of the effect.

Health care systems and local health economies always have unique features and disease prevalence, as well as different local barriers to optimal implementation of multidisciplinary programmes, and all are beset by the perennial problem of limited resources to get these programmes up and running. Nevertheless, there seem to be three critical elements:

- a trained heart failure nurse

- all the programmes that work include extensive education about heart failure for the patient, with regard to precipitating factors, ability to titrate diuretics to weight, dietary advice, and the need for compliance with medication

- access to clinicians trained in heart failure: in most of the studies the clinician was involved in writing the guidelines within which the nurse was to operate, and acted as a resource for the occasions when the nurse encountered problems outside of the protocol, providing advice and 
reassurance to the nurse, particularly regarding when the patient should be referred on to other health care professionals.

Both the American Heart Association and American College of Cardiology now recommend the use of multidisciplinary programmes of care for patients with heart failure. ${ }^{10}$ In addition, recent guidance to the National Health Service from the National Institute for Clinical Excellence has advised that heart failure care should be delivered as a multidisciplinary team with an integrated approach across the health care community. ${ }^{11}$

It is worth emphasising, however, that multiprofessional care is not just specialist nurse involvement in a vacuum but should truly be a team approach. While one individual offering optimal care can deliver excellent care to a small number of patients, if we are to deliver better health care for heart failure for the entire community then we have to utilise the team approach, which is capable of reaching a much wider audience.

\section{APPLYING THE MULTIDISCIPLINARY APPROACH TO MI}

Programmes of multidisciplinary care have been instituted after MI. In the USA, the cardiac hospitalisation atherosclerosis management programme (CHAMP) was a multidisciplinary hospital initiative involving nurses, doctors, medication advice, dietary advice, and exercise counselling after MI. ${ }^{12}$

Comparing treatment rates and outcome in the two year period before (1992-1993) and after (1994-1995) multidisciplinary care was instituted, the intervention resulted in a dramatic increase in the prescription of statins and aspirin. Importantly, these increased prescription rates were sustained over seven years and appeared to be associated with a reduction in events in terms of new infarcts, heart failure, hospitalisations, and total mortality.

So a multidisciplinary approach can be put into the postMI setting. However, there are a few caveats about post-MI heart failure and left ventricular dysfunction that need to be addressed. Despite the evidence from randomised trials showing the benefits of using ACE inhibitors, ${ }^{13-15} \beta$ blockers, ${ }^{16}$ and eplerenone ${ }^{17}$ in the post-MI setting, it is important to remember that all of these drugs were started within three days of the MI, usually in the coronary care unit, a highly specialised setting with trained cardiac nurses, doctors, and intensive monitoring. The initial up-titration was done in the coronary care unit or on the cardiac wards, and the follow up after up-titration was every three or four months thereafter for those on ACE inhibitors and $\beta$ blockers, and was even more intensive for those enrolled in the clinical trial examining the impact of eplerenone. So if we are to deliver heart failure care after MI to the standards of the clinical trials, then we have to bear in mind the degree of monitoring likely to be involved.

In terms of starting $\beta$ blockers, the correct place to do this is in hospital: in the post-MI situation, the CAPRICORN study demonstrated that this could be done safely in hospital. ${ }^{16}$ The importance of in-hospital initiation of drug treatments cannot be overstated. In the USA, the IMPACTHF trial showed that patients in whom a $\beta$ blocker was initiated in hospital after admission for decompensated heart failure were much more likely to remain on treatment at 60 days than those who were discharged without a $\beta$ blocker with a plan to start it in an outpatient setting. ${ }^{18}$ At 60 days, $91 \%$ of those initiated in hospital were still taking treatment compared with $73 \%$ of those initiated after discharge $(\mathrm{p}<0.0001)$.

A large study from the USA has shown that patients not discharged on $\beta$ blockers are unlikely to be started on them as outpatients. ${ }^{19}$ Among patients who were discharged on $\beta$ blockers, $85 \%$ of survivors had filled a prescription by 30 days post-discharge, and $61 \%$ were current users at 365 days. By contrast, only $8 \%$ of those patients with no discharge order for $\beta$ blockers had filled such a prescription by 30 days, and just $12 \%$ of patients were current users at one year.

Clearly, the characteristics of the post-MI situation are important: this is a structured setting within an acute unit in which there are integrated care pathways, access to specialist nurses, physicians, pharmacists, ability to monitor blood tests, and to monitor blood pressure. Importantly, the clinician has a patient who, at that point, is focused on his or her cardiovascular risk. Wherever possible, the clinician should seize the opportunity of that moment to create a link between the index event and the need for long term treatment.

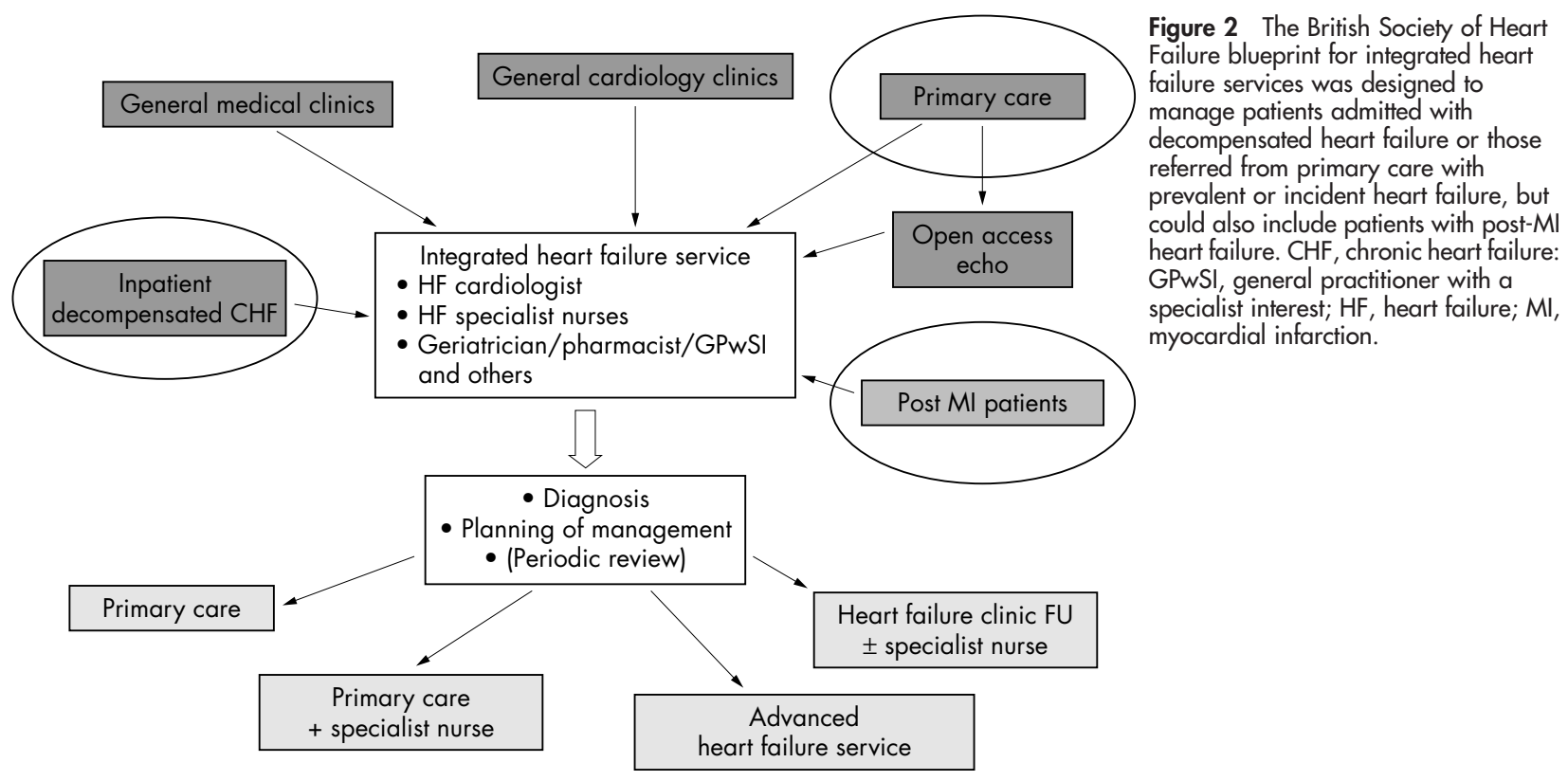

Figure 2 The British Society of Heart Failure blueprint for integrated heart failure services was designed to decompensated heart failure or those referred from primary care with prevalent or incident heart failure, but so include patients with post-MI heart failure. CHF, chronic heart failure: GPwSI, general practitioner with a specialist interest; $\mathrm{HF}$, heart failure; $M \mathrm{Ml}$ heart failure service 


\section{Learning points}

- Delivering evidence based care to patients with chronic heart failure has been a difficult and slow process

- Success came with the development of multidisciplinary heart failure management programmes

- A similar approach now needs to be adopted for managing patients with post-myocardial infarction heart failure

There appears to be something inherent in the post-MI situation that gives rise to worries about adherence. Data from the GRACE study confirm the high discontinuation of treatments in the first year after acute coronary syndrome admission: the discontinuation rate for $\beta$ blockers was $12 \%$ at the end of the year, and for ACE inhibitors it was 20\%. ${ }^{20}$

All of this demonstrates that it is not just prescribing medication that is important: this is a complex problem. Adherence with medication has much to do with the patient's beliefs about effectiveness of the treatment, their attitudes and ideas about side effects, and also with cost and dosage.

\section{CONCLUSION}

The post-MI situation is very complex. It is an acute situation in which clinicians are worrying about revascularisation, initiating many drugs and discharging the patient. In the UK, patients usually come back at six weeks to a multidisciplinary clinic, where they are again assessed by the clinician for ischaemia and left ventricular function, decisions have to be made about devices, and a rehabilitation programme is initiated.

Once all of these elements are taken care of, the clinician is often left with the patients who have heart failure and left ventricular dysfunction. How should they be managed? Rather than advocate post-MI specialist nurses, it should be remembered that this is just another part of the continuum of cardiovascular disease. Most patients who end up with heart failure start with an index MI, after which they develop left ventricular dysfunction and heart failure (fig l). It is not a different disease. The British Society of Heart Failure has developed a blueprint for clinical networks for integrated heart failure services across the UK (fig 2). At the moment, most nurse specialist programmes concentrate on picking up those patients admitted with decompensated heart failure or those referred from primary care with prevalent or incident heart failure. What many of these programmes exclude is the post-MI patient with heart failure and left ventricular dysfunction. The care of these patients needs to be integrated into our emerging multidisciplinary management programmes for heart failure.

\section{REFERENCES}

1 European Society of Cardiology. Task force for the diagnosis and treatment of chronic heart failure, European Society of Cardiology. Eur Heart $J$ 2001;22:1527-60.
2 Komajda M, Follath F, Swedberg K, et al for the Study Group on Diagnosis of the Working Group on Heart Failure of the European Society of Cardiology. The EuroHeart Failure Survey programme-a survey on the quality of care among patients with heart failure in Europe. Part 2: treatment. Eur Heart $J$ 2003:24:464-74.

3 Cleland JG, Cohen-Solal A, Aguilar JC, et al for the IMPROVEMENT of Heart Failure Programme Committees and Investigators. Improvement programme in evaluation and management; study group on diagnosis of the working group on heart failure of the European Society of Cardiology. Management of heart failure in primary care (the IMPROVEMENT of heart failure programme): an international survey. Lancet 2002;360:1631-9.

4 Spencer FA, Meyer TE, Gore JM, et al. Heterogeneity in the management and outcomes of patients with acute myocardial infarction complicated by heart failure: the national registry of myocardial infarction. Circulation 2002; 105:2605-10

5 Cowie MR, Murray-Thomas T, Underwood R, et al. Predictors and impact of worsening renal function in patients hospitalised with decompensated heart failure: a multi-centre European study. Eur Heart J 2004;25(abstract suppl):651 (abstract number 3750).

6 Rich MW, Beckham V, Wittenberg C, et al. A multidisciplinary intervention to prevent the readmission of elderly patients with congestive heart failure. N Engl J Med 1995;333:1190-5.

7 Stewart S, Marley JE, Horowitz JD. Effects of a multidisciplinary, home-based intervention on unplanned readmissions and survival among patients with chronic congestive heart failure: a randomised controlled study. Lancet 1999;354:1077-83.

8 Blue L, Lang E, McMurray JJ, et al. Randomised controlled trial of specialist nurse intervention in heart failure. BMJ 2001;323:715-8.

9 McAlister FA, Stewart S, Ferrua S, et al. Multidisciplinary strategies for the management of heart failure patients at high risk for admission: a systematic review of randomized trials. J Am Coll Cardiol 2004;44:810-9.

10 Hunt SA, Baker DW, Chin MH, et al. ACC/AHA guidelines for the evaluation and management of chronic heart failure in the adult: executive summary a report of the American College of Cardiology/American Heart Association task force on practice guidelines (committee to revise the 1995 guidelines for the evaluation and management of heart failure): developed in collaboration with the International Society for Heart and Lung Transplantation; endorsed by the Heart Failure Society of America. Circulation 2001;104:2996-3007.

11 National Institute for Clinical Excellence. Management of chronic heart failure in adults in primary and secondary care. Clinical Guideline 2003;5.

12 Fonarow GC, Gawlinski A, Moughrabi S, et al. Improved treatment of coronary heart disease by implementation of a cardiac hospitalization atherosclerosis management program (CHAMP). Am J Cardiol 2001;87:819-22.

13 Pfeffer MA, Braunwald E, Moye LA, et al. Effect of captopril on mortality and morbidity in patients with left ventricular dysfunction after myocardial infarction. Results of the survival and ventricular enlargement trial. The SAVE investigators. N Engl J Med 1992;327:669-77.

14 Kober L, Torp-Pedersen C, Carlsen JE, et al. A clinical trial of the angiotensin-converting-enzyme inhibitor trandolapril in patients with left ventricular dysfunction after myocardial infarction. Trandolapril cardiac evaluation (TRACE) study group. N Engl J Med 1995;333:1670-6.

15 The Acute Infarction Ramipril Efficacy (AIRE) Study Investigators. Effect of ramipril on mortality and morbidity of survivors of acute myocardial infarction with clinical evidence of heart failure. Lancet 1993;342:821-8.

16 Dargie HJ. Effect of carvedilol on outcome after myocardial infarction in patients with left-ventricular dysfunction: the CAPRICORN randomised trial. Lancet 2001;357:1385-90.

17 Pitt B, Remme W, Zannad F, et al for the Eplerenone Post-Acute Myocardial Infarction Heart Failure Efficacy and Survival Study Investigators. Eplerenone, a selective aldosterone blocker, in patients with left ventricular dysfunction after myocardial infarction. N Engl J Med 2003;348:1309-21.

18 Gattis WA, O'Connor CM, Gallup DS, et al for the IMPACT-HF Investigators and Coordinators. Predischarge initiation of carvedilol in patients hospitalized for decompensated heart failure: results of the initiation management predischarge: process for assessment of carvedilol therapy in heart failure (IMPACT-HF) trial. J Am Coll Cardiol 2004;43:1534-41.

19 Butler J, Arbogast PG, BeLue R, et al. Outpatient adherence to beta-blocker therapy after acute myocardial infarction. J Am Coll Cardiol 2002;40: 1589-95.

20 Eagle KA, Kline-Rogers E, Goodman SG, et al. Adherence to evidence-based therapies after discharge for acute coronary syndromes: an ongoing prospective, observational study. Am J Med 2004;117:73-81. 